\title{
COM OS USUÁRIOS EM MENTE: UM DESAFIO PARA A BOA PRÁTICA ARQUITETÔNICA?
}

\author{
WITH USERS IN MIND: A CHALLENGE FOR THE BEST ARCHITECTURE \\ PRACTICE?
}

\author{
Sheila Walbe Ornstein 1 \\ Universidade de São Paulo, São Paulo, SP, Brasil, sheilawo@usp.br
}

\begin{abstract}
Resumo
A arquitetura, a começar pela elaboração do programa de necessidades, deve atender aos usuários. Esse artigo busca discutir as formas e os instrumentos mais adequados à disposição dos arquitetos para atender aos usuários finais, a partir da concepção arquitetônica. Embora as interfaces objeto - usuário sejam bastante conhecidas dos designers no desenvolvimento dos produtos (objetos móveis e ou portáteis), na arquitetura - em que pese a interdisciplinaridade desta área do conhecimento - na prática, nem sempre estes projetistas conseguem alcançar o seu objetivo final. Considerando, no objetivo final, a satisfação do usuário associada à qualidade do produto o ambiente construído - nas suas dimensões formal e estética, funcional e técnica. Aqui são descritos e analisados, para os diversos ambientes construídos e em uso - em termos de escalas, usos coletivos e em termos daqueles voltados a grupos menores e mais homogêneos de usuários - os instrumentos disponíveis para o entendimento sobre a percepção, as necessidades e as expectativas dos usuários, com base nas pesquisas de Avaliação Pós-Ocupação (APO).
\end{abstract}

Palavras-chave: Usuários. Avaliação Pós-Ocupação. Instrumentos. Procedimentos metodológicos. Boa arquitetura.

\begin{abstract}
Architecture and its design process, starting with the brief, should support and attend users of the built environment. This article discusses suitable ways and tools available for architects to fulfill final user wishes, outlined in the first design sketches. Although the relation between product and user is well known to designers, in architecture, even taking into consideration that this field is based on an interdisciplinary approach, professionals are not always able to reach a goal of user satisfaction with the quality of the built environment and its formal-aesthetic and functional-technical dimensions. This article describes and analyses available tools to support the evaluation and understanding of the built environment of different types and scales from the perspective and perception of users and their needs, through Post-Occupancy Evaluation (POE) research.
\end{abstract}

Keywords: Users. Post-Occupancy Evaluation. Tools. Methodological procedures. Good architecture.

How to cite this article:

ORNSTEIN, Sheila Walbe. Com os usuários em mente: um desafio para a boa prática arquitetônica? PARC Pesquisa em Arquitetura e Construção, Campinas, SP, v. 7, n. 3, p. 189-197, out. 2016. ISSN 1980-6809. Disponível em:

<http://periodicos.sbu.unicamp.br/ojs/index.php/parc/article/view/8647437>. Acesso em: 17 mar. 2017.

doi:http://dx.doi.org/10.20396/parc.v7i3.8647437.

\section{Introdução}

A Avaliação Pós-Ocupação (APO), como área do conhecimento, vem sendo aplicada no Brasil desde meados dos anos 1980, em atividades de ensino (graduação e pós-graduação), pesquisa (iniciações científicas, mestrados e doutorados) e algumas atividades de consultoria. Os anais dos Encontros Nacionais de Tecnologia do Ambiente Construído ${ }^{1}$ (ENTAC) promovidos pela Associação Nacional de Tecnologia do Ambiente Construído (ANTAC), atestam que dezenas de pesquisas foram concluídas ou estão em curso nesta área no Brasil, sobretudo em Universidades públicas. Mais recentemente, temas vinculados a procedimentos metodológicos de APO com foco em acessibilidade, segurança contra incêndio e estudos de caso como as habitações (VILLA; ORNSTEIN, 2013) destinadas ao mercado imobiliário, hospitais, estações metroviárias, escolas, edifícios de escritórios, museus e outras ampliaram o leque de aplicações metodológicas e do potencial da APO e seus resultados para a realimentação de diretrizes de projeto. Wayfinding (CARPMAN; GRANT, 2002; ANDRADE; BINS ELY, 2014), mapas de fluxos e de comportamentos e diversos tipos de checklists têm sido elaborados como insumos para as melhorias ambientais (AUGUSTIN; COLEMAN, 2012; MALLORY-HILL; PREISER; WATSON, 2012). 
Entretanto, observa-se que apesar dos mais de trinta anos de realização de pesquisas em APO no Brasil, ainda há conhecimento limitado, ou mesmo equivocado, por parte de alguns agentes da cadeia produtiva da construção civil e na própria área acadêmica das bases conceituais e metodológicas da APO, fundamentadas na Psicologia Ambiental. Aqui, merece destaque a obra do Dr. Wolfgang F.E. Preiser, professor emérito da Universidade de Cincinnati, Ohio, EUA, falecido em 2016. A sua obra é digna de ser estudada e analisada com atenção e já nas primeiras leituras, por todos aqueles que queiram se dedicar a APO como área do conhecimento. Seus trabalhos, a iniciar pelo fundamental "Post-Occupancy Evaluation" (PREISER; RABINOWITZ; WHITE, 1988) e outros mais recentes como Preiser e Vischer (2005) e Mallory-Hill, Preiser e Watson (2012), são importantes para o entendimento da cronologia histórica internacional da APO e suas várias vertentes metodológicas, sempre exemplificadas com estudos de caso de âmbito prático. Prof. Preiser, arquiteto com formação em psicologia ambiental (Environmental Psychology) ainda inova e se transforma em pioneiro no tratamento do gerenciamento de facilidades e infraestrutura predial a partir de diagnósticos obtidos na aplicação da APO (PREISER, 1993; FEDERAL FACILITIES COUNCIL, 2001). O Prof. Preiser também foi importante ao perceber, com muita propriedade, o potencial da aplicação da APO nos estudos, pesquisas e na prática profissional nos campos da acessibilidade e do desenho universal (PREISER, OSTROFF, 2001). Zimring (2002) aborda enfaticamente o papel desse pesquisador e suas contribuições para o ensino e as pesquisas na APO.

No Brasil, o Prof. Preiser deixa um legado e bases acadêmicas sólidas neste campo, sobretudo na Faculdade de Arquitetura e Urbanismo da Universidade de São Paulo, mas também em inúmeras outras escolas de arquitetura e de engenharia do país (ORNSTEIN; ONO, 2010). Esse legado também se apresenta na ênfase dada à relevância da realimentação do processo de projeto a partir do conhecimento sistêmico dos ambientes em uso e na visão crítica em geral do processo de se fazer arquitetura.

As questões relevantes à pesquisa social aplicada, aproximam-se, no caso brasileiro, ainda que lentamente, das pesquisas em APO na área de arquitetura. Essas pesquisas necessitam de tratamentos específicos de dados, seja através da estatística clássica e desde a seleção amostral, até aquela que lida com as incertezas dos resultados, em particular decorrentes das respostas às perguntas dos questionários associadas a escalas de valores.

A busca, de modo mais sistêmico, de autorizações para a aplicação de instrumentos - ainda que não invasivos - na Plataforma Brasil (MINISTÉRIO DA SAÚDE, 2016) tem mostrado claramente não só a preocupação com o tema ética na pesquisa em geral, mas em especial a preocupação com a relação pesquisador-pesquisado e suas nuanças. Quanto a este aspecto, estuda-se cada vez mais, na APO as relações entre arquitetura, psicologia (sobretudo a ambiental) e antropologia. Neste contexto, perguntas surgem: Como tratar o sujeito coletivo numa APO? (LEFÉVRE; LEFÉVRE, 2003). O pesquisador em arquitetura pode se manter distante e "neutro" em relação ao usuário pesquisado em dado ambiente, ou as relações entre ambos são inexoravelmente imbricadas? (RHEINGANTZ; AZEVEDO; BRASILEIRO; ALCANTARA, 2009). A pesquisa mais adequada em APO é aquela centrada no usuário, no ambiente ou em ambas? (NORMAN, 2006). Desta forma, é essencial para o pesquisador em APO que queira oferecer uma efetiva contribuição a boa prática na arquitetura colocar em discussão tais temas.

\section{Instrumentos}

A seleção e a elaboração adequadas dos instrumentos fazem cada vez mais parte do planejamento da pesquisa já que é pré-requisito dos Comitês de Ética (Plataforma Brasil) apresentá-los previamente com vistas a solicitação de autorização para a realização da pesquisa. Além da autorização do dirigente responsável pelos ambientes estudos de caso, nos casos dos instrumentos (a) a (c) e, (e) a (g), (k) e (l), apresentados a seguir, é necessário no caso dos adultos e idosos que todos os participantes, considerando ações que exigem interações presenciais entre pesquisador-pesquisado, assinem o termo de livreconsentimento referente a autorização dos resultados da aplicação dos instrumentos para fins acadêmicos. No caso de crianças, faz-se necessário solicitar também o consentimento/autorização dos pais ou tutores. Certamente o pré-requisito inicialmente mencionado implica na elaboração de instrumentos adequadamente formulados, o que se configura num benefício para as pesquisas em APO contemporâneas. Dentre os instrumentos adotados na APO (ZEISEL, 2006; PINHEIRO, GÜNTHER, 2008; RHEINGANTZ; AZEVEDO; BRASILEIRO; ALCANTARA, 2009; VILLA; ORNSTEIN, 2013; BECHTEL; CHURCHMAN 2002) e que se inserem neste contexto, podemos citar: (a) Entrevistas individuais com pessoas-chave; (b) Entrevistas em grupo; (c) Grupos focais; (d) Poemas dos desejos; (d) Desenhos; (f) Questionários tradicionais; (g) Questionários associados a imagens ou figuras; (h) Checklist para "as built"; (i) Checklist para levantamento de eventuais patologias construtivas; (j) Walkthrough pelo pesquisador; (k) Walkthrough do pesquisador com pessoas-chave; (l) Walkthrough de vivência; (m) Medições; (n) Mapas de fluxos; (o) Mapas de vestígios; (p) Mapas de comportamentos; (q) Quadro síntese e (r) 
Mapas de diagnóstico e de recomendações. Estes instrumentos serão apresentados a seguir.

\section{Entrevistas individuais com pessoas-chaves (a)}

Entrevistas individuais, com pessoas-chaves exigem roteiros enxutos, gravados e transcritos se autorizados. Exigem também instrumentos qualitativos, mas que mesmo assim possibilitam categorizações de palavraschaves e tabulações. Oferecem riqueza de argumentos e informações mais especializadas, incluindo embasamento para a elaboração de formulários de questionários e de checklists de caráter técnico. Recomenda-se a realização de roteiro de perguntas, relacionadas aos objetivos da APO, para nortear a abordagem do entrevistador. Porém, a entrevista não deve exceder 30 minutos devidos aos tempos requeridos, na sequência, em termos de transcrição.

\section{Entrevistas em grupo (b)}

Exigem a formulação de roteiro de perguntas, destinada a um grupo de usuários específico e relacionada aos objetivos da APO. Podem ser realizadas por um único moderador (ao contrário do grupo focal que exige o moderador e um auxiliar). Podem ser realizadas com um grupo de 5 a 7 indivíduos. Também neste caso recomendase não exceder 30 minutos de duração já que também é feita a transcrição. $\mathrm{O}$ moderador preferencialmente deve ter alguma experiência com este tipo de entrevista e deve procurar ser um facilitador dos comentários e dos argumentos de todos os participantes, evitando perguntas e respostas concentradas num único participante, atuante como um eventual líder nesta atividade.

\section{Grupos focais (c)}

Tem as mesmas características e procedimentos da entrevista em grupo, porém requer além do moderador, um auxiliar que busca fazer anotações sobre atitudes e comportamentos dos participantes durante a realização desta ação e realizar registros fotográficos. O auxiliar também controla a operação do gravador. Dependendo do tipo de grupo focal, o auxiliar requerido tem formação em psicologia ambiental.

\section{Poemas dos desejos (d)}

Tem caráter exploratório e lúdico. Via de regra parte de um trecho de frase a ser completada pelo participante que relaciona a finalização da frase com a sua expectativa em relação ao presente ou ao futuro de um determinado edifício, ambiente, lugar. Por exemplo "eu gostaria que a minha sala de aula fosse......". Pode ser preenchido por crianças alfabetizadas e adultos. Os poemas são recolhidos periodicamente pelo pesquisador. Assim como nas entrevistas, as frases podem ser classificadas (ou palavraschaves) e quantitativamente organizadas em gráficos, por exemplo. Podem ser utilizados em ambientes como praças, museus ou escolas, dentre outros, cujos frequentadores /visitantes/ usuários esperam vivenciar ambientes diferenciados e que lhe ofereçam aprendizado de modo interativo.

\section{Desenhos (e)}

Têm um caráter exploratório, são aplicados individualmente em crianças não alfabetizadas ou mesmo semi-alfabetizados. Os desenhos (exercício de curta duração, não mais do que 10 minutos e no máximo dois desenhos sequenciais) partem de uma pergunta sobre um dado ambiente (presente e futuro) sobre a qual a criança deve responder no formato de um desenho (ELISEI; 2008; BECHTEL; CHURCHMAN, 2002). O pesquisador, ao término do exercício também pergunta a criança, o significado do desenho, para se certificar de sua compreensão. São realizados sobre papel branco, jogo de lápis coloridos. Se o pesquisador não tiver experiência prévia com este tipo de exercício, a presença e o acompanhamento de um psicólogo ou de um educador, é desejável. Para a aplicação deste instrumento, faz-se necessário um ambiente com cadeiras e mesas (preferencialmente individuais, evitando assim contato entre as crianças participantes durante a ação) adequadas para desenhos a serem feitos por crianças ou adultos. Neste caso o pesquisador oferece o papel (folhas em branco A4) e lápis coloridos.

É possível ainda considerar a possibilidade de adoção de protocolos de desenhos mais flexíveis e amigáveis, como os aplicativos de pintura digital ${ }^{2}$ com vistas ao aumento de interesse das crianças a partir do uso de tablets e a condução desta atividade de forma lúdica e menos rígida (brincadeiras).

\section{Questionários tradicionais (f)}

O questionário tradicional (HAYES, 1995; PINHEIRO; GÜNTHER, 2008) é o instrumento mais utilizado para medir a satisfação dos usuários adultos, pois apresenta baixo custo e é de aplicação relativamente rápida. Este instrumento requer realização de pré-teste para a aferição de sua inteligibilidade para leigos, o tempo para a sua aplicação e um desenho amostral coerente com o universo populacional bem como o objetivo da APO. Recomendase que a aplicação de cada questionário não requeira mais do que 10 minutos. Deve ser dividido em blocos: social (perfil do respondente) e aferição da satisfação sobre $o$ edifício (os diversos pavimentos e os setores desse). Para medir a satisfação dos usuários utiliza-se escalas ímpares ou pares de valores (neste caso, forçando tendência), para as quais, ao final, são atribuídos valores numéricos o que permite processamento de dados e transposição destes em gráficos. Predominam respostas fechadas e ou múltiplas escolhas, mas podem requerer também justificativas abertas (ainda que curtas) em relação a algumas respostas 
fechadas, ou espaço ao final para comentários abertos por parte do respondente. As escalas são necessariamente simétricas (para cada atributo "positivo" existe um atributo "negativo") e evita-se sobreposições de perguntas numa única, priorizando a objetividade e a precisão das questões. A elaboração, a aplicação e o processamento/tabulação de dados podem ser auxiliados por ferramentas computacionais ${ }^{3}$ associados a uso de tablets no campo. São aplicados nas diversas modalidades de edifícios, tais como estações metroviárias, museus, hospitais, bibliotecas, habitações e outras. As exigências de processamento e transformação em gráficos dos dados colhidos em campo, de forma cada vez mais rápida e com o menor número de vieses de tabulação também tem exigido que os pesquisadores utilizem as ferramentas do tipo daquelas acima mencionadas.

\section{Questionários associados a imagens ou figuras (g)}

Os questionários associados a imagens ou figuras, basicamente, devem seguir os mesmos procedimentos dos questionários tradicionais, porém são mais lúdicos e curtos pois estão associados a imagens. Por exemplo, de fachadas que se pretendem avaliar do ponto de vista da percepção e da apreciação visual do respondente, vinculando, como respostas atributos simétricos e totalmente opostos (a cada "positivo" corresponde um "negativo"). Este tipo de questionário (que pode ser apresentado ao respondente, de modo presencial num formulário convencional ou contemplando cartelas de figuras) pode ser particularmente interessante quando se pretende obter respostas sobre formas, cores e outras qualidades e características espaciais, formais e ou estéticas.

\section{Checklist para "as built" (h)}

O Checklist para "as built" é um roteiro para atualização dos ambientes, objeto da APO. A ação de atualização dos ambientes é fundamental neste tipo de pesquisa, já que os levantamentos são feitos com base em situações reais, em ambientes em uso (já contemplando seu mobiliário, usuários e infraestrutura).

\section{Checklist para levantamento de eventuais patologias construtivas (i)}

O Checklist para levantamento de eventuais patologias construtivas é desenvolvido com base na ABNT NBR 15575 (MEREB, 2015). Trata-se de um roteiro para realização de verificação a olho nu de possíveis patologias como infiltrações de águas de chuvas, desgastes de acabamento de pisos, caixilharia com funcionamento inadequado, armaduras expostas de elementos em concreto armado, fissuras em alvenarias e em seus revestimentos e outros (GOMIDE; FAGUNDES NETO; GULLO, 2015).

\section{Walkthrough pelo pesquisador (j)}

O Walkthrough a ser realizado pelo pesquisador segue um roteiro baseado em checklist sobre o sistema construtivo e seu desempenho (MEREB, 2015). Este instrumento requer o percurso detalhado no interior dos ambientes e junto aos itens exteriores do edifício (por exemplo, fachadas, coberturas, itens relacionados à segurança contra incêndio e outros). Sob esta perspectiva, drones poderiam ser utilizados para tornar esta ação mais ágil e precisa.

\section{Walkthrough do pesquisador com pessoas-chaves} (k).

A ser realizado pelo pesquisador com especialistas como o arquiteto que concebeu o edifício estudo de caso, o engenheiro responsável pela manutenção e operação, o técnico responsável pela segurança (contra incêndio, patrimonial), dentre outros, com base num roteiro. $\mathrm{O}$ roteiro segue o formato de entrevista a ser feita no decorrer do percurso, o que possibilitaria a identificação, pela pessoa chave/especialista, de aspectos específicos de cada ambiente.

\section{Walkthrough de vivência (I)}

Walkthrough com pessoas com deficiência (visual ou em cadeira de rodas) é também chamado de vivência. Desenvolve-se a partir de um percurso a ser feito com base num roteiro/checklist prévio junto com pessoas com deficiência (ideal) ou em simulações, ou seja, um dos pesquisadores poderia fazer o percurso em cadeira de rodas e numa outra oportunidade com uma venda nos olhos. Estes walkthroughs têm por objetivo observar as eventuais dificuldades e ou barreiras das pessoas com deficiência nos ambientes. Estes walkthroughs podem ser também realizados com idosos ou com pesquisadores "vestidos" com kits de envelhecimento (estudos ergométricos se possível com o auxílio de gerontólogos).

\section{Medições (m)}

As medições com equipamentos portáteis na área de conforto ambiental (térmica, iluminação, umidade, acústica) devem seguir as normas para cada tipo de medição.

\section{Mapas de fluxos (n)}

Os mapas de fluxo são bastante úteis em edifícios complexos, de grande porte e destinados a múltiplos usuários (THOMAZONI; ORNSTEIN; ONO, 2016). Exigem a leitura dos projetos (atualizados, "as built") e as observações sistematizadas no tempo (dias e horários) dos fluxos das diversas categorias de usuários e de materiais. Os mapas de fluxos permitem a visualização de conflitos em áreas de circulação em função da disposição de ambientes em pavimentos e no edifício. Este é um 
procedimento muito pertinente para grandes hospitais e arenas esportivas, por exemplo.

\section{Mapas de vestígios (o)}

Mapas de vestígios são observações sistemáticas considerando dias e horários de uso dos ambientes, sobretudo aqueles de uso coletivo, como pátios de recreio em escolas, parques e praças, áreas externas a campi universitários. Estas observações identificam os percursos mais utilizados pelas pessoas (indivíduos, grupos) a partir de vestígios encontrados como papéis, restos de alimentos, caminhos espontâneos em áreas gramadas e outros.

\section{Mapas de comportamentos (p)}

Os mapas de comportamento são observações sistemáticas de comportamentos de usuários, de modo individual ou coletivo em distintos ambientes, dias e horas (ZEISEL, 2006; PINHEIRO; GÜNTHER, 2008).

\section{Quadro síntese (q)}

O Quadro Síntese compõe a base do diagnóstico de especialistas ou pesquisadores versus usuários. São planilhas eletrônicas que sintetizam os temas de APO tratados, os instrumentos utilizados, os cruzamentos "especialistas versus usuários", os principais resultados, as recomendações, as normas de desempenho vinculadas, as prioridades de intervenção em função de nível de risco envolvido e eventuais observações. O Quadro Síntese é fundamental para a visão geral do conjunto dos resultados da $\mathrm{APO}$ e para a definição de quais aspectos (boas práticas a serem melhoradas) devem ser inseridos nos mapas de diagnósticos e de recomendações.

\section{Mapas de diagnóstico e de recomendações ( $r$ )}

Parte dos itens listados no Quadro Síntese são transportados para estes mapas que são baseados nos desenhos dos diversos pavimentos do edifício (incluindo áreas externas e vizinhança, muitas vezes) aos quais são apontados, a partir de linhas de chamada, os aspectos mais relevantes encontrados na APO, suas recomendações e normas vinculadas. Nestes mapas há legendas com níveis de risco e cronograma estimado para implementação das ações (curto, médio e longo prazos, em função dos riscos). Podem ser muito úteis também aos gestores de facilidades ou de uso, operação e manutenção.

\section{Discussão}

Como é possível depreender, a partir do rol de instrumentos voltados às medições do desempenho físico dos ambientes e da satisfação dos usuários, há um arsenal de procedimentos que podem ser adotados pelos pesquisadores - e projetistas - para entender melhor as relações "ambiente construído versus comportamento" com vistas a qualificar com mais propriedade, o programa de necessidades e o que os usuários efetivamente esperam da concepção arquitetônica (KOWALTOWSKI et al., 2011).

Os instrumentos descritos e analisados neste artigo podem e devem ser utilizados de forma combinada, dependendo da natureza da pesquisa, dos temas a serem abordados e do perfil dos estudos de caso. Destaca-se também que os (i), (j) e (m) são levantamentos físicos a serem realizados pelo pesquisador, especialista em APO, e que antecedem a aplicação dos instrumentos focados nos usuários (todos os demais) pois considera-se essencial a atualização dos desenhos arquitetônicos ("as built") e a compreensão das condições físicas dos ambientes, antes de se iniciar os estudos relativos às relações Ambiente ConstruídoComportamento Humano (Environmental Psychology/Environment and Behavior). Isto se dá pela necessidade, no caso brasileiro, de se ter elementos físicos e gráficos confiáveis e atualizados no tempo para, então, se proceder a etapa da APO sob a ótica do usuário. E neste momento - anterior a verificação das necessidades e expectativas dos usuários - é que se verifica o quanto os projetos e os ambientes em uso, atendem ao quadro normativo (desempenho físico) e legislativo edilício vigentes, o que deve ou deveria ocorrer, independentemente das expectativas e do atendimento às necessidades dos usuários. No caso específico dos instrumentos (m), estes poderão ser aplicados ou não, se o tema da pesquisa em APO tiver como prioridade os aspectos de conforto ambiental. Porém, os demais, (i) e (j) são essenciais para se iniciar uma APO. Já o instrumento (r) é a representação visual baseada no quadro síntese, instrumento (q), anterior dos diagnósticos e das recomendações decorrentes da APO ("especialistas versus usuários) e, por isto, sugere-se a sua elaboração, já que facilita a utilização dos resultados da APO pelos tomadores de decisão, por exemplo, os gestores de facilidades.

As pesquisas em Avaliação Pós-Ocupação foram significativamente ampliadas no Brasil, nas últimas décadas quanto a diversidade de estudos de casos (desde residências, até hospitais, aeroportos, museus, zoológicos e outros equipamentos urbanos) (BITGOOD, 2002) e de procedimentos metodológicos experimentados (PINHEIRO; GÜNTHER, 2008; IMMS; CLEVELAND; FISHER, 2016; PREISER; VISHER, 2005; KOWALTOWSKI et al., 2013).

Entretanto, ainda há muito o que fazer. Nesta direção, podemos mencionar:

(i) A necessidade do treinamento do olhar do pesquisador sobre os ambientes e sobre os seus usuários (VOORDT; WEGEN, 2005); 
(ii) A necessidade da efetiva realização de pesquisas com equipes interdisciplinares, envolvendo não só arquitetos como engenheiros, mas também designers, psicólogos, antropólogos, educadores e outros. Alguns grupos de pesquisadores em APO realizam tal aproximação, porém considerando a ampla disseminação do tema - avaliação de desempenho em uso, pode-se dizer que são iniciativas ainda incipientes;

(iii) Maior rigor com o tratamento e a modelagem estatística de dados (CORRAL-VERDUGO, 2002; VITTORINO; ONO, 2013) sobretudo em relação a definição de amostra de usuários respondentes de questionários e de aplicação de outros instrumentos e de descritores estatísticos a serem utilizados (margem de erro, nível de confiabilidade, correções e outros;

(iv) Ampliação consistente dos estudos e pesquisas de APO relacionados a outras modelagens no campo da estatística "não clássica" como a lógica fuzzy (MORAES, 2008) e que permitiria outras considerações diferenciadas sobre as incertezas das respostas (questionários) de usuários quando as questões estão associadas a escalas de valores e flutuações de tendências "para o positivo" ou "para o negativo";

(v) Submissão, como prática do cotidiano da pesquisa, desta e seus instrumentos, na Plataforma Brasil, o que está associado aos aspectos de ética na abordagem de seres humanos nos estudos. Este procedimento, no campo das ciências sociais aplicadas, pode ser relativamente simples, uma vez que não existem abordagens "invasivas" ou eventuais malefícios à saúde de usuários e pode ser útil e proteger não só os respondentes e entrevistados, mas também o próprio pesquisador;

(vi) Ampliar as pesquisas sobre o sujeito coletivo (LEFÉVRE; LEFÉVRE, 2003; BARBOSA, 2015), para o caso de ambientes "de massa" com estações de metrô, aeroportuárias, arenas, parques e outros;

(vii) ampliar, com vistas a redução dos tempos necessários para os levantamentos de campo, sem prejuízo deste, aspecto fundamental na APO (obtenção de dados reais, em campo), a utilização em determinados contextos, de simulações e jogos (IDEO, 2016; DELIBERADOR; KOWALTOWSKI, 2015) os quais podem auxiliar se combinados com respostas de aplicações concretas;

(viii) Ampliar pesquisas sobre ferramentas que auxiliem na automação dos cruzamentos de dados "especialistas/pesquisadores versus usuários" para geração de diagnósticos (VILLA; SARAMAGO; CASASANTA, 2015; VILLA et al., 2016). Nesta direção, é possível que o Building Modeling Information (BIM), a gestão de bancos de dados (FRANÇA; ORNSTEIN, 2016; SALES; RUSCHEL, 2014) e o Geographic Information System (GIS) possam trazer colaborações importantes.
Especialmente quanto ao GIS ou Sistema de Informações Geográficas (SIG), segundo Golledge (2002) e Lima e Lay (2012), procedimentos computacionais podem converter informações geográficas - urbanas e arquitetônicas - em dados úteis para a análise e a avaliação da cognição espacial de usuários, como proximidade, dispersão, mobilidade, conectividade, formas, percursos, segurança, além de sobrepor ou não em $2 \mathrm{D}$ e em $3 \mathrm{D}$ camadas de diferentes tipos de usos urbanos, como o comercial, o residência, equipamentos voltados à saúde, educacionais e outros;

(ix) Ampliar os estudos e pesquisas sobre a análise e a avaliação das relações ambiente construído versus comportamento humano, a partir da sintaxe espacial, conforme apontam Peponis e Wineman (2002) e na esteira da extensa obra de Hillier (HILLIER; HANSON, 1984), sobretudo nos anos 1979 e 1980, combinando, por exemplo, as leituras gráficas oriundas da sintaxe espacial com mapas de fluxos e observações sistêmicas de comportamentos de usuários;

(x) Ampliar os estudos e pesquisas sobre "custos das recomendações e das intervenções oriundas dos resultados das pesquisas em APO". Este é um campo de pesquisa que possui ainda muitos enfrentamentos já que o cliente do projeto de arquitetura e da obra construída poderá ter interesse na APO se esta for concluída com dados objetivos de "custos versus benefícios";

(xi) Finalmente, ampliar o oferecimento de treinamento a arquitetos e urbanistas para que sejam destacados os programas de necessidades formulados a partir da compreensão das relações "ambiente construído versus comportamento humano" conforme aponta Hershberger (2002).

\section{Considerações finais}

A APO se propõe a fornecer insumos a pesquisadores em arquitetura e urbanismo e a projetistas para que o resultado da concepção arquitetônica atenda às necessidades e satisfaça não só o cliente, mas também ao usuário final, à luz de cada tipologia de edifício. Para tanto, as variáveis são múltiplas e o arquiteto deve fazer escolhas e definir prioridades que atendem não só aos anseios dos futuros usuários, mas a um conjunto significativo de aspectos técnicos, funcionais e legais.

O desafio para os projetistas está em obter um equilíbrio entre os itens de maior relevância que precisa necessariamente atender na sua prática profissional e, certamente, o usuário. Nesta direção, o entendimento do comportamento e das necessidades do usuário é a essência de sua atuação, para que ocorra a boa prática arquitetônica. A pesquisa no campo da APO - método científico com 
vistas à realimentação do processo de projeto - pode colaborar com este tipo de encaminhamento.

\section{Notas}

(1) http://www.infohab.org.br/entac/

(2) Um exemplo de aplicativo para pintura digital é o Fresh Paint para o Windows da Microsoft (https://www.microsoft.com/pt-br/store/p/fresh-paint/9wzdncrfjb13, acessado em 14.03.2017).

(3) Dois exemplos são: (i) as soluções online (em nuvem) para colaboração da Goolgle como Documentos, Formulários e Planilhas e (ii) os aplicativos para pesquisa e coleta de dados off-line como o QuickTapSurvey (http://www.quicktapsurvey.com/lg/pt/, acessado em 29.10.16).

\section{Referências}

ANDRADE, Isabela, F.; BINS ELY, Vera Moro. Orientação espacial em terminal aeroportuário: diferentes perspectivas. In: III ENCONTRO DA ASSOCIAÇÃO NACIONAL DE PESQUISA E PÓS-GRADUAÇÃO EM ARQUITETURA E URBANISMO. São Paulo. Anais....São Paulo, ANPARQ, 2014. Disponível em www.anparq.org.br/ Acesso em 26.02.2017.

AUGUSTIN, Sally; COLEMAN, Cindy. The Designer's Guide to Doing Research. Applying knowledge to Inform Design. Hoboken, New Jersey: John Wiley \& Sons, Inc, 2012. 302p.

BARBOSA, Maria Beatriz Pestana. Wayfinding na jornada da pessoa com deficiência visual no sistema metroferroviário. 2015. Tese (Doutorado em Tecnologia da Arquitetura) - Faculdade de Arquitetura e Urbanismo, Universidade de São Paulo, São Paulo, 2015. Disponível em: <http://www.teses.usp.br/teses/disponiveis/16/16132/tde-08032016-163338/>. Acesso em: 01.11.2016.

BECHTEL, Robert B.; CHURCHMAN, Arza. (eds). Handbook of Environment Psychology. 1a edição. New York: John Wiley \& Sons, 2002. 722p.

BITGOOD, Stephen C. Environment Psychology in Museus, Zoos, and other Exhibition Centers. In: Bechtel, R.B.; Churchman, A. (eds). Handbook of Environment Psychology. 1a edição. New York: John Wiley \& Sons, 2002. pp. 461-480.

CARPMAN, Janet R; GRANT, Myron, A. Wayfinding: A Broad View. In: BECHTEL, R.B.; CHURCHMAN, A. (eds). Handbook of Environment Psychology. 1a edição. New York: John Wiley \& Sons, 2002. pp. 427-442.

GOLLEDGE, Reginald, G..The Open Door of GIS. In: BECHTEL, R.B.; CHURCHMAN, A. (eds). Handbook of Environment Psychology. 1a edição. New York: John Wiley \& Sons, 2002. pp. 244-255.

CORRAL-VERDUGO, Victor. Structural Equation Modeling. In: BECHTEL, R.B.; CHURCHMAN, A. (eds). Handbook of Environment Psychology. 1a edição. New York: John Wiley \& Sons, 2002. pp.256-270.

DELIBERADOR, Marcella Savioli; KOWALTOWSKI, Doris Catharine Cornelie Knatz. O jogo como ferramenta de apoio ao programa arquitetônico de escolas públicas. PARC Pesquisa em Arquitetura e Construção, Campinas, SP, v. 6, n. 2, p. 85-102, jun. 2015. ISSN 1980-6809. doi:http://dx.doi.org/10.20396/parc.v6i2.8634985.

ELISEI, Mateus Guedes Martins. Diagnóstico da percepção ambiental através do desenho infantil. Taubaté, SP: Cabral Editora e Livraria Universitária, 2008. 95p.

FEDERAL FACILITIES COUNCIL. Learning from our buildings. A State-of-the-Practice Summary of Post-Occupancy Evaluation. Washington, DC: The National Academy Press, 2001. 129p. Disponível em https://www.nap.edu/read/10288/chapter/ Acesso em 28.02.2017.

FRANÇA, Ana Judite Galbiatti Limongi; ORNSTEIN, Sheila Walbe. Modelagem da informação e melhoria contínua do ambiente construído: oportunidades e desafios. In: ENCONTRO NACIONAL DE TECNOLOGIA DO AMBIENTE CONSTRUÍDO. 16., 2016, São Paulo. Anais ... São Paulo: ANTAC, 2016. p. 4834-4848. Diponível em: http://www.infohab.org.br/entac/2016/ENTAC2016_paper_536.pdf. Acesso em: 31.10.2016 
GOMIDE, Tito Livio Ferreira; FAGUNDES NETO, Jerônimo Cabral P.; GULLO, Marco Antonio. Engenharia Diagnóstica em Edificações. São Paulo: Pini, 2015. 424p.

HAYES, Bob H. Medindo a satisfação do cliente. Desenvolvimento e uso de questionários. Rio de Janeiro: Qualitymark, 1995. 209 p.

HERSHBERGER, Robert. Behavioral-Based Architectural Programming. In: BECHTEL, R.B.; CHURCHMAN, A. (eds). Handbook of Environment Psychology. 1a edição. New York: John Wiley \& Sons, 2002. pp. 292-305.

HILLIER, Bill; HANSON, Julienne. The social logic of space. Cambridge: Cambridge: Cambridge University Press, 1984. 281p.

IDEO. Methods Cards. Disponível em: https://www.ideo.com/post/method-cards. Acesso em: 29.10.2016

IMMS, Wesley; CLEVELAND, Benjamin; FISHER, Kenn (Ed.). Evaluating Learning Environments. Snapshots of Emerging Issues, Methods and Knowledge. Rottherdam, The Netherlands: Sense Publishers, 2016. 257p.

KOWALTOWSKI, D. K. et al. (eds.). O processo de projeto em arquitetura: da teoria à tecnologia. São Paulo, Brasil: Oficina de Textos, 2011. 504p.

KOWALTOWSKI, Doris C.C.K. et al. Métodos e instrumentos de avaliação de projetos destinados à habitação de interesse social. In: VILLA S.B; ORNSTEIN, S.W. (orgs). Qualidade ambiental na habitação. Avaliação Pós-Ocupação. São Paulo: Oficina de Textos, 2013. pp.149-184.

LEFÉVRE, Fernando; LEFÉVRE, Ana Maria Cavalcanti. O discurso do sujeito coletivo. Um novo enfoque em pesquisa qualitativa (desdobramentos). Caxias do Sul, RS: EDUCS, 2003. 255p.

LIMA, Márcia Azevedo de; LAY, Maria Cristina Dias. A Configuração de conjunto habitacionais e seus efeitos na interação social. Arquisur, n. 2, 2012. pp. 72-87. Disponível em http://bibliotecavirtual.unl.edu.ar/ojs/index.php/ARQUISUR/article/view/933/1394. Acesso em 27.02.2017.

MALLORY-HILL, Shauna; PREISER, Wolfgang F.E.; WATSON, Chris (Ed.). Enhancing Building Performance. Iowa, USA: Wiley-Blackwell, 2012. 330p.

MEREB, Marcia Pellegrini (Coord.) Guia para arquitetos na aplicação da Norma de Desempenho ABNT NBR 15575. São Paulo: ASBEA, 2015, p.56. Disponível em: http://www.caubr.gov.br/wp-content/uploads/2015/09/2 guia normas final.pdf. Acesso em: 25.10.2016

MINISTÉRIO DA SAÚDE. Plataforma Brasil. Disponível em: http://aplicacao.saude.gov.br/plataformabrasil/login.jsf . Acesso em: 26.10.2016.

MORAES, Odair Barbosa de. Método de análise de dados para avaliação de áreas urbanas recuperadas - uma abordagem utilizando a lógica fuzzy. 2008. Tese (Doutorado em Engenharia de Construção Civil e Urbana) - Escola Politécnica, Universidade de São Paulo, São Paulo, 2008. doi: http://dx.doi.org/10.11606/T.3.2008.tde-17112008-120123.

NORMAN, Donald A. O design do dia a dia. Rio de Janeiro: Rocco, 2006. 271p.

ORNSTEIN, Sheila Walbe; ONO, Rosaria. Post-Occupancy Evaluation and Design Quality in Brazil: Concepts, Approaches and an Example of Application. Gestão \& Tecnologia de Projetos, Brasil, v. 5, n. 1, p. 138-138, may 2010. ISSN 1981-1543. doi:http://dx.doi.org/10.4237/gtp.v5i1.159..

PEPONIS, John; WINEMAN, Jean. Spatial Structure of Environment and Behaviour. In: BECHTEL, R.B.; CHURCHMAN, A. (eds). Handbook of Environment Psychology. 1a edição. New York: John Wiley \& Sons, 2002. pp. 271-291

PINHEIRO, José de Queiroz; GÜNTHER, Hartmut (Orgs.). Métodos de Pesquisa nos Estudos Pessoa-Ambiente. São Paulo: Casa do Psicólogo, 2008. 396p.

PREISER, Wolfgang F.E.; VISCHER, Jacqueline C. (Eds.). Assessing Building Performance. Oxford, UK: Elsevier, 2005. 243p. 
PREISER, Wolfgang F.E.; RABINOWITZ, Harvey, Z; WHITE, Edward. Post-Occupancy Evaluation. New York: Van Nostrand Reinhold, 1988. 198p.

PREISER, Wolfgang F.E. Professional practice in facility programming. New York: Van Nostrand Reinhold, 1993. 541p.

PREISER, Wolfgang F.E.; OSTROFF, Elaine. Universal Design Handbook. New York: Mc Graw Hill, 2001.

RHEINGANTZ, Paulo Afonso; AZEVEDO, Giselle Arteiro; BRASILEIRO, Alice; ALCANTARA, Denise de; QUEIROZ, Mônica. Observando a qualidade do lugar, Procedimentos para a avaliação pós-ocupação. Rio de Janeiro: ProArq / UFRJ, 2009. 117p. Disponível em: http://www.fau.ufrj.br/prolugar/assets/obs_a_qua_lugar.pdf Acesso em: 25.10.2016.

SALES, Adriano de Alencar; RUSCHEL, Regina C. BIM no suporte a Avaliação Pós-Ocupação . In: ENCONTRO NACIONAL DE TECNLOGIA DO AMBIENTE CONSTRUÍDO, 15., Fortaleza. Anais....Fortaleza: ANTAC, 2014. http://doi.org/10.17012/entac2014.6.

THOMAZONI, Andrea D’Angelo Leitner; ORNSTEIN, Sheila Walbe; ONO, Rosaria. Post-Occupancy Evaluation applied to design of a complex hospital by means of flow analysis. In: REVISITING THE ROLE OF ARCHITECTURAL SCIENCE IN DESIGN \& PRACTICE. 50 ${ }^{\mathrm{TH}}$ INTERNATIONAL CONFERENCE OF THE ARCHITECTURAL SCIENCE ASSOCIATION. 2016, Adelaide, Australia. Proceedings....Adelaide, Australia:ASA, 2016. p. 537- 546.

VILLA, Simone Barbosa; SARAMAGO, Rita de Cássia Pereira; GARCIA, Lucianne Casasanta, Avaliação pós-ocupação no Programa Minha Casa Minha Vida. Uma experiência metodológica. Uberlândia: UFU/ PROEX, 2015, 152p. Disponível em: https://morahabitacao.files.wordpress.com/2015/07/os-014631-proex-ufu-livro-sangria-lu.pdf. Acesso em: 25.10.2016.

VILLA, Simone Barbosa; ORNSTEIN, Sheila Walbe. (Orgs). Qualidade Ambiental na Habitação. Avaliação Pós-Ocupação. São Paulo: Oficina de Textos, 2013. 400p.

VILLA, Simone Barbosa et al.. Inovação tecnológica na avaliação pós-ocupação: ferramentas digitais e interativas. In: ENCONTRO NACIONAL DE TECNOLOGIA DO AMBIENTE CONSTRUÍDO. 16., 2016, São Paulo. Anais ... São Paulo: ANTAC, 2016. p. 3439-3452. Disponível em: http://www.infohab.org.br/entac/2016/ENTAC2016_paper_263.pdf. Acesso em: 31.10.2016

VITTORINO, Fulvio; ONO, Rosaria. Técnicas estatísticas aplicadas à APO em habitações. In: VILLA, Simone Barbosa; ORNSTEIN, Sheila Walbe. (Orgs). Qualidade Ambiental na Habitação. Avaliação Pós-Ocupação. São Paulo: Oficina de Textos, 2013. pp.185-206.

VOORDT, Theo JM van der; WEGEN, Herman BR van. Architecture in use. An Introduction to programming, design and evaluation of buildings. Oxford, UK: Elsevier, 2005. 237p.

ZEISEL, John. Inquiry by design. Environment /Behavior/ Neuroscience in Architecture, Interiors, Landscape, and Planning. New York: W.W. Norton \& Company Ltd, 2006. 399p.

ZIMRING, Craig. Postoccupancy Evaluation: Issues and Implementation. In: BECHTEL, R.B.; CHURCHMAN, A. (eds). Handbook of Environment Psychology. 1a edição. New York: John Wiley \& Sons, 2002. pp. 306-319.

\footnotetext{
${ }^{1}$ Sheila Walbe Ornstein

Arquiteta Urbanista. Professora Titular da Faculdade de Arquitetura e Urbanismo da Universidade de São Paulo. Bolsista Produtividade do Conselho Nacional de Desenvolvimento Científico e Tecnológico (CNPq). Endereço postal: Rua do Lago, 876, Cidade Universitária, Butantã, São Paulo, SP, Brasil, CEP 05508-080.
} 